\title{
Hereditary cryohydrocytosis with reduced stomatin
}

INSERM

\section{Source}

INSERM. (1999). Orphanet: an online rare disease and orphan drug data base. Hereditary cryohydrocytosis with reduced stomatin. ORPHA:168577

Hereditary cryohydrocytosis with reduced stomatin is a rare hemolytic anemia

characterized by combination of neurologic features, such as psychomotor delay, seizures, variable movement disorders, and hemolytic anemia with stomatocytosis, resulting in cation-leaky erythrocytes, pseudohyperkalemia, hemolytic crises and hepatosplenomegaly. Cataracts are also a presenting feature. 